Special issue in honor of Prof. George C. Papageorgiou

\title{
Limiting steps and the contribution of alternative electron flow pathways in the recovery of the photosynthetic functions after freezing-induced desiccation of Haberlea rhodopensis
}

\author{
K. GEORGIEVA* (D) A.V. POPOVA* (D), G. MIHAILOVA* (D), A.G. IVANOV***** (D), \\ and M. VELITCHKOVA ${ }^{* *++}$
}

Institute of Plant Physiology and Genetics, Bulgarian Academy of Sciences, Acad. G. Bonchev Str., Bl. 21, 1113 Sofia, Bulgaria*

Institute of Biophysics and Biomedical Engineering, Bulgarian Academy of Sciences, Acad. G. Bonchev Str., Bl. 21, 1113 Sofia, Bulgaria**

Department of Biology, University of Western Ontario, 1151 Richmond Str. N, London, N6A 5 B7 Ontario, Canada**

\begin{abstract}
Haberlea rhodopensis Friv. is unique with its ability to survive desiccation to an air-dry state during periods of extreme drought and freezing temperatures. To understand its survival strategies, it is important to examine the protective mechanisms not only during desiccation but also during rehydration. We investigated the involvement of alternative cyclic electron pathways during the recovery of photosynthetic functions after freezing-induced desiccation. Using electron transport inhibitors, the role of PGR5-dependent and NDH-dependent PSI-cyclic electron flows and plastid terminal oxidase were assessed during rehydration of desiccated leaves. Recovery of PSII and PSI, the capacity of PSI-driven cyclic electron flow, the redox state of plastoquinone pool, and the intersystem electron pool were analyzed. Data showed that the effect of alternative flows is more pronounced in the first hours of rehydration. In addition, the NDH-dependent cyclic pathway played a more determining role in the recovery of PSI than in the recovery of PSII.
\end{abstract}

Keywords: alternative electron flow; chlorophyll fluorescence; cyclic electron flows; freezing-induced desiccation; rehydration.

\section{Introduction}

Haberlea rhodopensis Friv. belongs to the group of desiccation-tolerant or resurrection plants that can survive desiccation to an air-dry state. Upon rehydration, they rapidly revive and restore their metabolic activity (Moore et al. 2009). The characteristic feature of H. rhodopensis is its ability to withstand freezing temperatures during

\section{Highlights}

- During rehydration of Haberlea rhodopensis, PSI recovered earlier than PSII

- Recovery of photosynthetic performance is mediated by PGR5- and

NDH-dependent CEF

- PTOX-dependent electron transfer to oxygen is involved in the recovery process
Received 27 September 2021 Accepted 16 February 2022

Published online 7 March 2022

Corresponding author e-mail: mayav@bio21.bas.bg

\footnotetext{
Abbreviations: $\left(1-\mathrm{F}_{\mathrm{v}}{ }^{\prime} / \mathrm{F}_{\mathrm{m}}{ }^{\prime}\right)$ - relative proportion of the energy absorbed and dissipated as heat in the PSII antennae; $\left(1-\mathrm{q}_{\mathrm{P}}\right)-$ excitation pressure of PSII; AD - distilled water; AntA - antimycin A; CEF - cyclic electron flow; Chl - chlorophyll; FR - far-red; $F_{v} / F_{m}-$ maximum photochemical efficiency of PSII; FQR - ferredoxin:plastoquinone oxidoreductase; LEF - linear electron transport; MT - multiple turnover flash; NDH - chloroplast NADPH dehydrogenase-like complex; NPQ - nonphotochemical quenching; OG - octyl gallate; $\mathrm{P}_{700}, \mathrm{P}_{700}{ }^{+}$- reduced and oxidized reaction center chlorophyll of PSI; PGR5 - proton gradient regulation 5; PQ - plastoquinone; $\mathrm{R}_{\mathrm{fd}}-\mathrm{Chl}$ fluorescence decrease ratio; RWC - relative water content; ST - single turnover flash; WWC - water-water cycle; $\Phi_{\mathrm{PSII}}-$ actual photochemical efficiency of PSII.

Acknowledgments: This work was supported by the Bulgarian National Science Fund, Ministry of Education and Science (Project КП-06-H21/8).

Conflict of interest: The authors declare that they have no conflict of interest.
} 
winter. Similar to drought, freezing stress causes also dehydration of plants; they survive the harsh winter conditions in a dry state (Mihailova et al. 2020). Our previous results revealed that exposure of $H$. rhodopensis to temperatures below $-6^{\circ} \mathrm{C}$ induces ultrastructural changes in mesophyll cells comparable to desiccation. However, while the complete rearrangement in the cell commences at 20\% RWC under drought stress (Georgieva et al. 2017), it started at the beginning of freezing when the RWC was above 60\% (Mihailova et al. 2020). Although a significant part of chloroplasts remained intact during freezing, major rearrangements in the abundance/ organization of the main photosynthetic pigment-protein complexes were observed (Sárvári et al. 2014). In addition, increased thermal energy dissipation, downregulation of photosynthesis, and high antioxidant activity enable plants to overcome oxidative stress during chilling and freezing temperatures (Mihailova et al. 2020, Georgieva et al. 2021).

Desiccation tolerance can be achieved either by mechanisms of protection of cellular integrity or by mechanisms of repair of desiccation or rehydrationinduced cellular damage (Bewley and Oliver 1992). These mechanisms are part of the survival strategy of resurrection plants and must be coordinated to permit successful recovery from desiccation. Most of the studies on resurrection plants have focused on the characterization of protection mechanisms in the dehydration phase, while the rehydration process has been scarcely investigated (Gao and Wang 2012, Gao et al. 2013, Giarola and Bartels 2015). Since at the cellular level, the chloroplasts play a dual role, as a sensor of various environmental cues in some acclimation responses, and as a major target of environmentally induced structural and functional changes (Hüner et al. 2016), the response of the photosynthetic performance during desiccation-rehydration cycles in resurrection plants is of major interest.

As a result of light reactions of photosynthesis, the electron transfer between electron transport carriers occurred in the thylakoid membranes resulting in accumulation of NADPH and generation of proton gradient utilized during synthesis of ATP. The electron transport from water in PSII to $\mathrm{NADP}^{+}$mediated by photosynthetic electron transport carriers (PSII, PSI, cytochrome $b_{6} / f$, plastoquinone, plastocyanin) is named linear electron transport. In addition, cyclic electron transport pathways around PSI depend solely on the PSI photochemical performance (Shikanai 2007 and references therein). In higher plants, two main partially redundant routes of cyclic electron transfer were recognized - antimycin A (AntA)-sensitive (PGR5-dependent) CEF (Arnon et al. 1954) and NDH-dependent (Shikanai et al. 1998). PSI-dependent CEF mediated by the chloroplast NDH complex is different from the classical version. Although Arnon's pathway is sensitive to AntA (Tagawa et al. 1963), the NDH complex is resistant to the same concentration of AntA (Endo et al. 1997). It is well documented that under abiotic stress conditions, CEF is stimulated to compensate imbalance between the effectiveness of light reactions and metabolic processes. It has been suggested that although the two CEF pathways are partially redundant, each of them may fulfill different functions: the NDH-dependent pathway may serve predominantly as a safety valve that prevents over-reduction of the stroma, while the PGR5dependent PSI CEF is essential and mostly involved in maintaining the correct production ratio of ATP/NADPH (Shikanai 2007). The significant role of CEFs in response to cold stress has been widely discussed for Arabidopsis thaliana (Ivanov et al. 2012) and maize (Savitch et al. 2011). There is relatively scarce data on the involvement of CEF in plant drought and especially in rehydration. The effect of cyclic electron flow during desiccation and brief rehydration of the red alga Porphyra yezoensis has been reported (Gao and Wang 2012).

Our recent studies on the recovery of photosynthetic activity during rehydration of $H$. rhodopensis from drought- and freezing-induced desiccation showed that PSI activity recovered faster compared to PSII (Georgieva et al. 2020). Furthermore, restoration of PSI maximum activity was reached after $24 \mathrm{~h}$ of rehydration, when PSII activity significantly increased. Thus, the contribution of alternative electron flow pathways in the recovery of PSI activity was suggested. Earlier, the significance of enhanced PSI-dependent CEF for the survival of Ulva sp. (Chlorophyta) in desiccated conditions (Gao et al. 2011) and the recovery from severe desiccation of intertidal macro-alga Porphyra yezoensis (Gao and Wang 2012) and Porphyra haitanensis (Gao et al. 2013) have been reported. However, studies addressing the potential role of $\mathrm{CEF}$ and other electron transport pathways in alleviating the functional limitations during desiccation-rehydration cycles in resurrection terrestrial plants species are very limited and controversial (Huang et al. 2012, FloresBavestrello et al. 2016).

In the present study, the contribution of alternative electron flow pathways in the recovery of the photosynthetic activity during rehydration of Haberlea rhodopensis plants after freezing-induced desiccation was assessed by using specific electron transport inhibitors of PGR5-dependent (AntA) and $\mathrm{NDH}$-dependent $\left(\mathrm{HgCl}_{2}\right)$ cyclic electron pathways, as well as, PTOX-mediated (octyl-gallate, OG) electron flow to oxygen (Savitch et al. 2011, Ivanov et al. 2012). Recovery of photosynthetic competence of PSII was determined by analysis of chlorophyll (Chl) fluorescence by using of PAM fluorometer and that of PSI by determination of redox state of $\mathrm{P}_{700}$.

\section{Materials and methods}

Plant material: Haberlea rhodopensis Friv. tufts of shade ecotype were initially collected from the Rhodope Mountains and further cultivated in pots with peat soil (Stender, Schermbeck, Germany) under ex situ (outdoor) environmental conditions. Plants were exposed to cold and freezing temperatures during autumn and winter (November-February). The light intensity during the experiment was $30-60 \mu \mathrm{mol}\left(\right.$ photon) $\mathrm{m}^{-2} \mathrm{~s}^{-1}$. Low positive temperatures did not influence the relative water content (RWC) of leaves. But when the temperature dropped to 
about $-10^{\circ} \mathrm{C}$, the dehydration started and plants were in an air-dry state during the winter. Leaf discs from dry plants were used for rehydration in distilled water or the solutions of specific electron transport inhibitors. The experiment was performed in March when the rehydration started under natural conditions.

Treatment during rehydration: Discs from selected leaves from desiccated to the air-dried state Haberlea rhodopensis plants were soaked in distilled water or the solutions of studied inhibitors at the designated concentration for different periods - for 1, 3, 5, 7, and $24 \mathrm{~h}$ under illumination with light intensity corresponding to the intensity during growth. The preliminary experiments showed that within $24 \mathrm{~h}$ the leaves became fully hydrated reaching an RWC of about $90 \%$. Three discs were measured at every time point for characterization of recovery of activities of PSI (by measuring $\mathrm{P}_{700}$ ) and PSII (by determination of parameters of $\mathrm{Chl}$ fluorescence using PAM fluorometer). The experiment was repeated twice.

For rehydration of leaf discs in the solutions of different inhibitors, the following concentrations were used: $\mathrm{HgCl}_{2}-100 \mu \mathrm{M}$; antimycin A $-10 \mu \mathrm{M}$; octyl gallate $20 \mu \mathrm{M}$.

Pulse-modulated Chl $\boldsymbol{a}$ fluorescence: Chl $a$ fluorescence induction was measured with a portable fluorometer PAM-2500 (Heinz Walz GmbH, Effeltrich, Germany). The leaf discs were dark-adapted for $15 \mathrm{~min}$ and PAR of $90 \mu$ mol(photon) $\mathrm{m}^{-2} \mathrm{~s}^{-1}$ was used for the measurements. The temperature during measurements was $21-23^{\circ} \mathrm{C}$. All used basic parameters were given by PamWin-3 software (Heinz Walz GmbH, Effeltrich, Germany). The actual efficiency of PSII electron transport during illumination was estimated at steady state as $\Phi_{\text {PSII }}=$ $\left(\mathrm{F}_{\mathrm{m}}{ }^{\prime}-\mathrm{F}_{\mathrm{s}}\right) / \mathrm{F}_{\mathrm{m}}{ }^{\prime}$ (Genty et al. 1989), where $\mathrm{F}_{\mathrm{m}}{ }^{\prime}$ is the maximum fluorescence and $F_{s}$ is the steady-state fluorescence in the light-adapted state. The ratio of fluorescence decrease $\left(\mathrm{F}_{\mathrm{d}}=\mathrm{F}_{\mathrm{m}}-\mathrm{F}_{\mathrm{s}}\right)$ to steady-state fluorescence was calculated as $R_{F d}=F_{d} / F_{S}$ (Lichtenthaler and Rinderle 1988). Excitation pressure of PSII, which gives an approximate measure of the reduction state of the first electron acceptor $\mathrm{Q}_{\mathrm{A}}$ of PSII, was calculated as $1-\mathrm{q}_{\mathrm{P}}$, as $\mathrm{q}_{\mathrm{P}}$ is determined by the equation $\mathrm{q}_{\mathrm{P}}=\left(\mathrm{F}_{\mathrm{m}}{ }^{\prime}-\mathrm{F}_{\mathrm{s}}\right) /\left(\mathrm{F}_{\mathrm{m}}{ }^{\prime}-\mathrm{F}_{0}\right)$ (van Kooten and Snel 1990). The relative proportion of the energy absorbed and dissipated as heat in the PSII antennae (referred to as thermal energy dissipation in the antenna) was estimated by $1-\left(\mathrm{F}_{\mathrm{v}}{ }^{\prime} / \mathrm{F}_{\mathrm{m}}{ }^{\prime}\right)$ (Demmig-Adams et al. 1996).

For determination of the reduction state of the plastoquinone pool, the post-illumination transient increase of Chl fluorescence at the $\mathrm{F}_{0}{ }^{\prime}$ level was followed (Asada et al. 1993, Mano et al. 1995).

Determination of photooxidable $\mathbf{P}_{700}$ : The redox state of $\mathrm{P}_{700}$ was monitored in vivo as $\Delta \mathrm{A}_{820-860}$ absorption changes. A Walz ED 700DW-E emitter/detector unit was connected to a PAM $101 \mathrm{E}$ main control unit (Heinz Walz $G m b H$, Effeltrich, Germany). $\mathrm{P}_{700}$ was oxidized by far-red (FR) light from a photodiode (FR-102, Heinz Walz GmbH,
Effeltrich, Germany). The intensity of FR light was $13.4 \mathrm{~W} \mathrm{~m}^{-2}$. FR light was controlled by the PAM 102 unit and applied via the multibranched fiber-optic system. The measurement was carried out in the reflection mode.

Transient reduction of $\mathbf{P}_{700}$ : The changes of $\mathrm{P}_{700}$ during rehydration of Haberlea rhodopensis leaves were followed using a PAM-101/103 modulated fluorometer (Heinz Walz $G m b H$, Effeltrich, Germany) equipped with an ED-800T emitter-detector unit (Klughammer and Schreiber 1991) as described in Ivanov et al. (1998). Discs from desiccated leaves were dark-adapted for $15 \mathrm{~min}$ before the start of measurements which were performed at $22^{\circ} \mathrm{C}$ and ambient $\mathrm{O}_{2}$ and $\mathrm{CO}_{2}$ conditions. Leaves were illuminated with far-red light $\left(\lambda_{\max }=715 \mathrm{~nm}, 13.4 \mathrm{~W} \mathrm{~m}^{-2}\right.$, Schott filter $R G$ 715). The redox state of $\mathrm{P}_{700}$ was registered as the FR-induced absorbance change around $820 \mathrm{~nm}\left(\Delta \mathrm{A}_{820}\right)$ in a custom-designed cuvette.

The capacity for CEF around PSI was estimated by the half time $\left(\tau_{1 / 2}\right)$ of the decay kinetics of re-reduction of $\mathrm{P}_{700}{ }^{+}$ after switching off the FR illumination (Klughammer and Schreiber 1991, Ivanov et al. 1998).

For estimation of apparent intersystem electron pool size (Asada et al. 1993, Savitch et al. 2011, Ivanov et al. 2012), the transient reduction of $\mathrm{P}_{700}{ }^{+}$after application of single turnover (ST, half peak $14 \mu \mathrm{s}$ ) and multiple turnover flashes (MT, $50 \mathrm{~ms}$ ) of saturating white light were applied by XMT-103 and XST-103 (Heinz Walz GmbH, Effeltrich, Germany) power/control units, respectively. The ratio between the area of MT to the area of ST flashes $\left(\mathrm{e}^{-} / \mathrm{P}_{700}=\mathrm{MT}\right.$ area/ST area $)$ represents the relative functional pool size of the intersystem electrons able to reduce PSI center $\left(\mathrm{P}_{700}{ }^{+}\right)$. The areas of ST and MT were determined as the complimentary area between the oxidation trace of $\mathrm{P}_{700}$ after either ST or MT pulse excitation and the stationary level of $\mathrm{P}_{700}{ }^{+}$under FR illumination (Asada et al. 1993, Ivanov et al. 1998).

Statistical analysis: Two rehydration cycles were performed for each inhibitor. The measurements of Chl $a$ fluorescence and $\Delta \mathrm{A}_{820}$ absorption changes were repeated three times per cycle using leaves from different plants $(n=6)$. Comparison of means was made by the Fisher's least significant difference (LSD) test at $P \leq 0.05$ following analysis of variance (ANOVA). A statistical software package (StatGraphics Plus, version 5.1 for Windows, USA) was used. Pearson's correlation coefficient $(r)$ was used to measure the strength of a linear association between two variables. It was calculated in Microsoft Excel. The formulas return a value between -1 and 1 , where 1 indicates a strong positive relationship and -1 indicates a strong negative relationship.

\section{Results}

Effect of inhibitors on the recovery of PSII activity during rehydration: The recovery of photochemical activity of PSII during rehydration of dry $H$. rhodopensis leaves in distilled water (AD) and in the presence of different electron transport inhibitors is presented in 


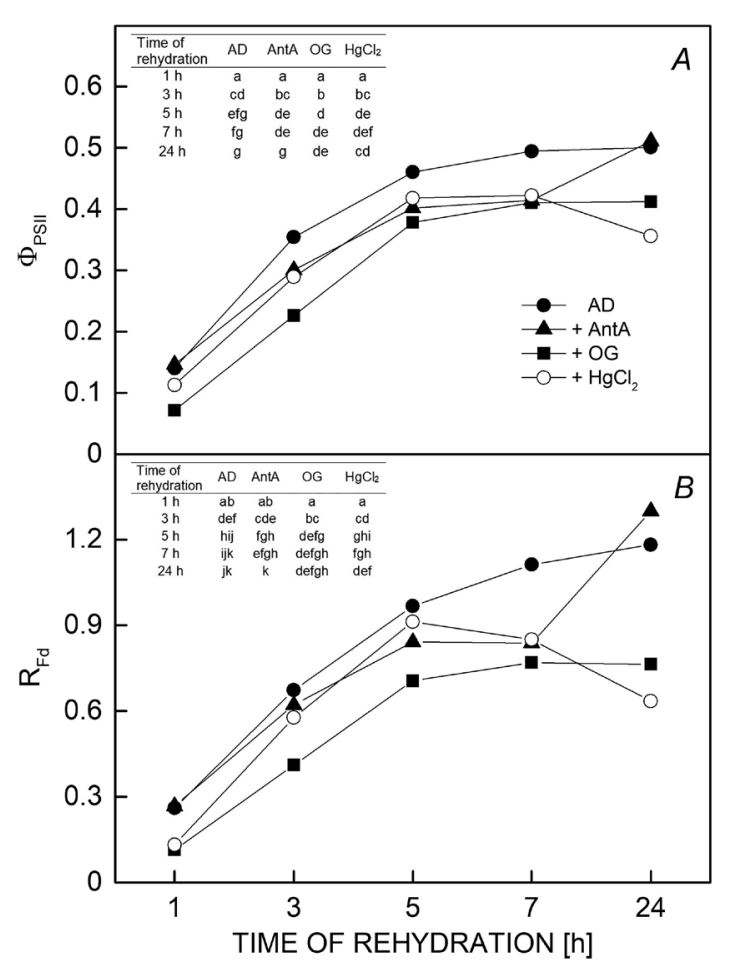

Fig. 1. Effects of electron transport inhibitors on the quantum yield of PSII photochemistry, $\Phi_{\text {PSII }}(A)$ and the vitality index, $\mathrm{R}_{\mathrm{Fd}}(B)$ in dehydrated Haberlea rhodopensis leaf discs during rehydration. Leaf discs were rehydrated for different periods $(1,3$, 5,7 , and $24 \mathrm{~h}$ ) either in distilled water (AD) or in AD containing $100 \mu \mathrm{M} \mathrm{HgCl} 2,10 \mu \mathrm{M}$ antimycin A (AntA), or $20 \mu \mathrm{M}$ octyl gallate (OG). The data are averages from three measurements in two independent experiments $(n=6)$. Insert represents the results from ANOVA statistical analyses; the same letters indicate no significant differences assessed by the Fisher's least significant difference (LSD) test at $P \leq 0.05$. All values represent mean values $\pm \mathrm{SE}$.

Fig. 1A. The quantum efficiency of electron transport, $\Phi_{\text {PSII }}$, which was completely inhibited in dry leaves, showed fast recovery reaching maximum value after $24 \mathrm{~h}$ of rehydration when leaves regain their RWC. This parameter is proportional to the product of photochemical quenching $\left(\mathrm{q}_{\mathrm{P}}\right)$ and the efficiency of excitation capture by open PSII reaction centers $\left(\mathrm{F}_{\mathrm{v}}{ }^{\prime} / \mathrm{F}_{\mathrm{m}}{ }^{\prime}\right)$ (Genty et al. 1989). The recovery of $\Phi_{\text {PSII }}$ during rehydration of dry leaves in $\mathrm{AD}$ was due to both the increased proportion of open PSII centers $\left(\mathrm{q}_{\mathrm{P}}\right)$ and the efficiency of their excitation capture (data not shown). Indeed, the high correlation coefficient of Pearson was determined for the rehydration induced changes in $\Phi_{\mathrm{PSII}}$ and $\mathrm{q}_{\mathrm{P}}(r=0.983)$ and $\mathrm{F}_{\mathrm{v}}{ }^{\prime} / \mathrm{F}_{\mathrm{m}}{ }^{\prime}$ $(r=0.998)$. The rehydration of dry discs in the presence of AntA, inhibiting PGR5-dependent PSI CEF, and $\mathrm{HgCl}_{2}$, inhibiting $\mathrm{NDH}$ complex, slightly decreased the $\Phi_{\mathrm{PSII}}$ values but the differences were statistically significant only for 24-h rehydration in the presence of $\mathrm{HgCl}_{2}$ (Fig. 1A). Similar changes were observed in the values of the ratio of Chl $a$ fluorescence decrease to the steady-state Chl $a$ fluorescence $\left(\mathrm{R}_{\mathrm{Fd}}\right)$ but they were better expressed compared to $\Phi_{\mathrm{PSII}}$ (Fig. $1 B$ ). $\mathrm{R}_{\mathrm{Fd}}$ covers the whole process of photosynthesis, including the full induction period, the transition of the photosynthetic apparatus from the nonfunctional state 1 to its functional state 2 , and also the photosynthetic $\mathrm{CO}_{2}$ fixation (Lichtenthaler and Rinderle 1988, Lichtenthaler and Miehé 1997). Thus, $R_{F d}$ values permit a fast screening of the photosynthetic activity and vitality of plants under stress. The results clearly showed that rehydration in the presence of octyl gallate (OG) that inhibits the plastid terminal oxidase (PTOX), significantly reduced the $\mathrm{R}_{\mathrm{Fd}}$ values (Fig. $1 B$ ).

The values of excitation pressure (estimated by $1-\mathrm{q}_{\mathrm{p}}$ ) were close to 1 in an air-dry state (Fig. $2 A$ ) and its values significantly decreased even after $1 \mathrm{~h}$ of rehydration in $\mathrm{AD}$ but remained higher in the presence of all inhibitors used. However, with increasing the photochemical activity of PSII, the values of $1-\mathrm{q}_{\mathrm{P}}$ decreased in the course of rehydration of dry leaves in the other inhibitors (Fig. 2A). Similar to excitation pressure, the relative proportion of the energy absorbed and dissipated as heat in the PSII

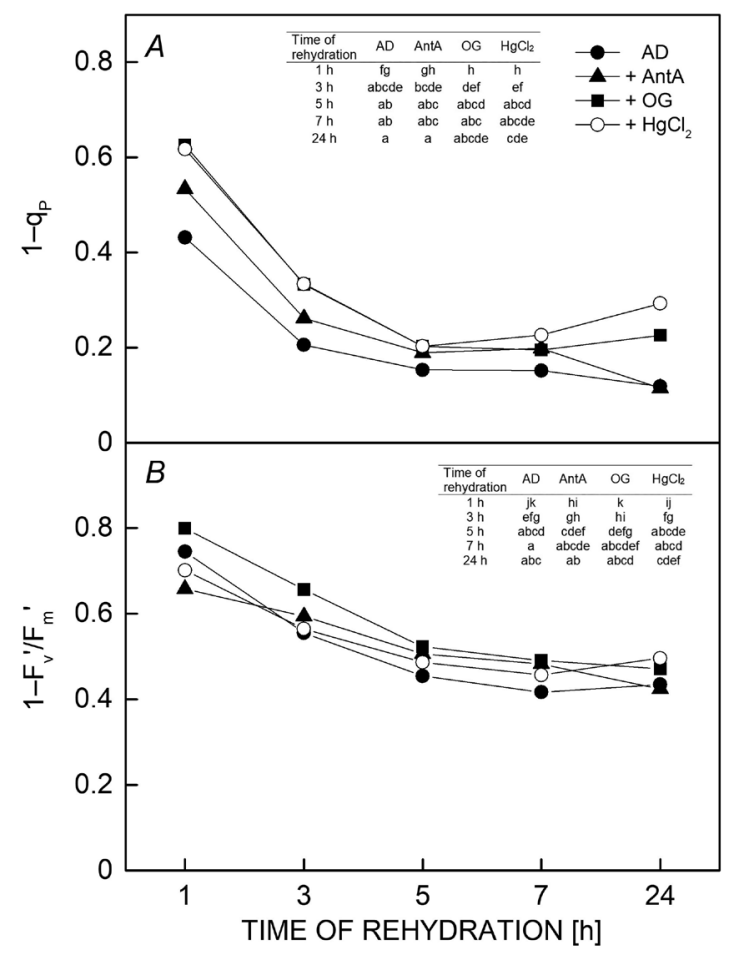

Fig. 2. Effects of electron transport inhibitors on the relative reduction state of PSII measured as $\left(1-\mathrm{q}_{\mathrm{P}}\right)(A)$ and the relative proportion of the energy absorbed and dissipated as heat in the PSII antennae estimated as $1-\left(\mathrm{F}_{\mathrm{v}}{ }^{\prime} / \mathrm{F}_{\mathrm{m}}{ }^{\prime}\right)(B)$ in dehydrated Haberlea rhodopensis leaf discs during rehydration. Leaf discs were rehydrated for different periods $(1,3,5,7$, and $24 \mathrm{~h})$ either in distilled water (AD) or in $\mathrm{AD}$ containing $100 \mu \mathrm{M} \mathrm{HgCl}$, $10 \mu \mathrm{M}$ antimycin $\mathrm{A}$ (AntA), or $20 \mu \mathrm{M}$ octyl gallate $(\mathrm{OG})$. The data are averages from three measurements in two independent experiments $(n=6)$. Insert represents the results from ANOVA statistical analyses; the same letters indicate no significant differences assessed by the Fisher's least significant difference (LSD) test at $P \leq 0.05$. All values represent mean values $\pm \mathrm{SE}$. 
antennae $\left(1-\mathrm{F}_{\mathrm{v}}{ }^{\prime} / \mathrm{F}_{\mathrm{m}}{ }^{\prime}\right)$ decreased as a result of rehydration in the presence of the other inhibitors (Fig. 2B).

Recovery of PSI during rehydration in presence of inhibitors: The effect of different inhibitors on the recovery of PSI activity was assessed by measuring the extent of FR light-induced absorbance change at $820 \mathrm{~nm}$ $\left(\Delta \mathrm{A}_{820-860}\right)$, which reflects the oxidation of $\mathrm{P}_{700}$ to $\mathrm{P}_{700}{ }^{+}$ (Fig. 3A). Fast enhancement in the activity of PSI was observed even after $1 \mathrm{~h}$ of rehydration of dry leaf discs in AD. In contrast to PSII, which activity increased upon rehydration up to $24 \mathrm{~h}$ (Fig. $1 A$ ), the values of $\Delta \mathrm{A}_{820-860}$ reached a maximum after $3 \mathrm{~h}$ of rehydration, remained unchanged until $7 \mathrm{~h}$, and slightly decreased after $24 \mathrm{~h}$. Comparison of the recovery of PSII and PSI activity in AD showed faster recovery of PSI, which was confirmed by a comparatively low correlation coefficient of Pearson for the rehydration induced changes in $\Phi_{\text {PSII }}$ and $\Delta \mathrm{A}_{820}$ $(r=0.584)$. In addition, the effects of different electron transport inhibitors were more pronounced on PSI activity compared to PSII. The recovery of photochemical activity

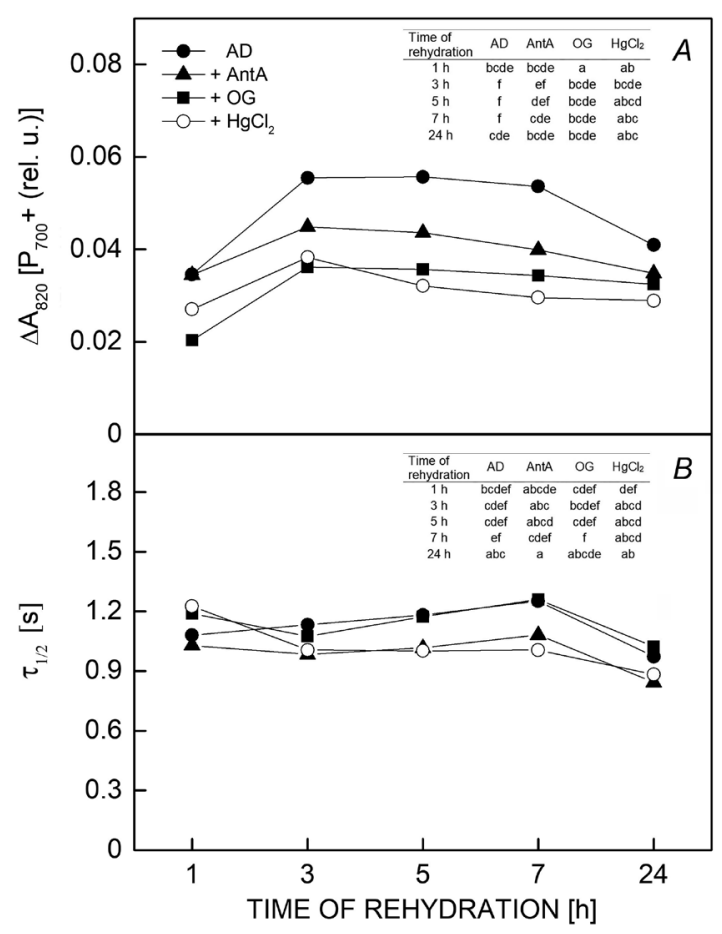

Fig. 3. Effects of electron transport inhibitors on the steady-state levels of far-red (FR) light-induced $\mathrm{P}_{700}$ photooxidation $\left(\mathrm{P}_{700^{+}}\right)$ measured by $\Delta \mathrm{A}_{820-860}(A)$ and half-times of $\mathrm{P}_{700}{ }^{+}$re-reduction $\left(\tau_{1 / 2}\right)$ after turning off the FR light illumination $(B)$ in dehydrated Haberlea rhodopensis leaf discs during rehydration. Leaf discs were rehydrated for different periods $(1,3,5,7$, and $24 \mathrm{~h})$ either in distilled water (AD) or in $\mathrm{AD}$ containing $100 \mu \mathrm{M} \mathrm{HgCl}$, $10 \mu \mathrm{M}$ antimycin $\mathrm{A}$ (AntA), or $20 \mu \mathrm{M}$ octyl gallate (OG). The data are averages from three measurements in two independent experiments $(n=6)$. Insert represents the results from ANOVA statistical analyses; the same letters indicate no significant differences assessed by the Fisher's least significant difference (LSD) test at $P \leq 0.05$. All values represent mean values \pm SE. of PSI was less affected by AntA than by the other inhibitors. A significant reduction in PSI activity was observed in the presence of $\mathrm{OG}$ and $\mathrm{HgCl}_{2}$ (Fig. $3 A$ ).

When the far-red light was turned off, the kinetics of the subsequent $\mathrm{P}_{700}{ }^{+}$decay in the dark are presumed to reflect primarily the rates of cyclic electron transport around PSI (Maxwell and Biggins 1976, Ravenel et al. 1994) and/or the interaction of stromal components with the intersystem electron transport chain (Asada et al. 1992). The rates of $\mathrm{P}_{700}{ }^{+}$re-reduction were 3-fold faster in dry leaves during the whole time interval of rehydration measured and regardless of inhibitors used during rehydration compared to control nondehydrated plants $\left(\tau_{1 / 2}=2.97 \pm 0.32\right.$ for control plants), thus indicating a higher capacity for CEF around PSI in desiccated plants and during the rehydration (Fig. 3B).

Estimation of intersystem electron pool and $P Q$ pool reduction: In Fig. $4 A$, the typical trace of $\mathrm{P}_{700}$ oxidationreduction transients demonstrating the effects of ST and MT flashes are presented. The application of ST flash of white light caused a fast chemical reduction of the steadystate level of FR-induced $\mathrm{P}_{700}{ }^{+}$followed by fast reoxidation back to the steady-state level of $\mathrm{P}_{700}{ }^{+}$. This represents the transient electron flow from PSII, thus giving a relative measure for functional PSII complexes (Losciale et al. 2008, Ivanov et al. 2012). Application of MT resulted in an almost full reduction of $\mathrm{P}_{700}{ }^{+}$caused not only by electrons produced in PSII, but also by electrons donated to the intersystem electron pool from the stroma (Asada et al. 1992, 1993). The ratio between areas of MT and ST represented the apparent functional electron $\left(\mathrm{e}^{-}\right)$pool size, i.e., number of $\mathrm{e}^{-}$per PSI (Fig. $4 B$ ). The maximum level of intersystem electrons that can be donated to PSI for leaf discs rehydrated over pure water was reached after $5 \mathrm{~h}$ of rehydration. In the presence of AntA, the maximum was reached at 3 and $5 \mathrm{~h}$, for $\mathrm{OG}$ at $7 \mathrm{~h}$, while for $\mathrm{HgCl}_{2}$, the maximum was observed after $3 \mathrm{~h}$ of rehydration. Not surprisingly, considering the limited capacity for LEF (Fig. 1A), the size of the intersystem electron pool was 3- to 4-fold lower in rehydrated leaf discs regardless of inhibitors used during the time course of rehydration compared to nondesiccated leaves $\left(\mathrm{e}^{-} / \mathrm{P}_{700}=\right.$ $7.52 \pm 0.82, n=11$ ).

The extent of dark-reduction of PQ pool by stroma reductants and/or intersystem electron pool was evaluated by recorded post-illumination increase of $\mathrm{F}_{0}{ }^{\prime}$ transients for $100 \mathrm{~s}$. Measurements were performed on freezingdesiccated leaf discs of $H$. rhodopensis after rehydration for different periods either over AD or over AD in presence of different electron transport inhibitors (Fig. 5). For leaf discs, rehydrated for $1 \mathrm{~h}$ in $\mathrm{AD}$, after $60 \mathrm{~s}$, a transient overreduction of the $\mathrm{PQ}$ pool was detected; it was significantly reduced after $5 \mathrm{~h}$ of rehydration and additionally reduced after $24 \mathrm{~h}$ of rehydration (Fig. $5 \mathrm{~A}$ ).

For leaf discs, rehydrated for $1 \mathrm{~h}$ in the presence of electron inhibitors, the extent of dark-induced reduction of PQ pool proceeded with a much slower rate - the reached level after $90 \mathrm{~s}$ represented $73 \%$ of the transient from $\mathrm{F}_{\mathrm{s}}$ to $\mathrm{F}_{0}{ }^{\prime}$ for AntA, and $27 \%$ for $\mathrm{OG}$ and $\mathrm{HgCl}_{2}$. 

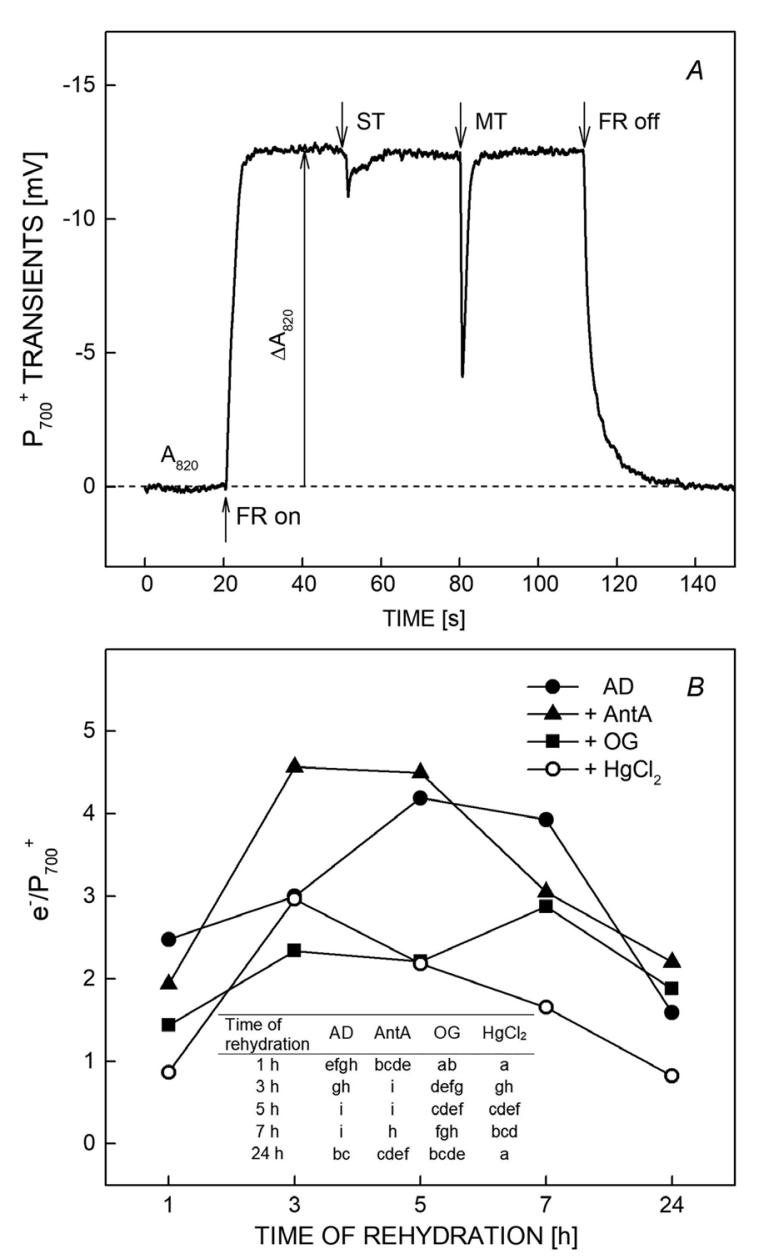

Fig. 4. Typical trace of far-red (FR) light-induced $\mathrm{P}_{700}$ transients measured at $820 \mathrm{~nm}$. After reaching a steady-state level of $\mathrm{P}_{700}$ oxidation $\left(\mathrm{P}_{700}{ }^{+}\right)$by FR illumination, single-turnover flash (ST) and multiple turnover flash (MT) pulses of white saturating light were applied $(A)$. Effects of electron transport inhibitors on the apparent electron donor pool size to PSI $\left(\mathrm{e}^{-} / \mathrm{P}_{700^{+}}\right)$as a function of the rehydration time of Haberlea rhodopensis leaf discs $(B)$. Leaf discs were rehydrated for different periods $(1,3,5,7$, and $24 \mathrm{~h}$ ) either in distilled water (AD) or in AD containing $100 \mu \mathrm{M}$ $\mathrm{HgCl}_{2}, 10 \mu \mathrm{M}$ antimycin A (AntA), or $20 \mu \mathrm{M}$ octyl gallate (OG). The data are averages from three measurements in two independent experiments $(n=6)$. Insert represents the results from ANOVA statistical analyses; the same letters indicate no significant differences assessed by the Fisher's least significant difference (LSD) test at $P \leq 0.05$.

For the longer period of rehydration in the presence of inhibitors, $5 \mathrm{~h}$, the level of PQ pool reduction increased in comparison with $1 \mathrm{~h}$ of rehydration. After $24 \mathrm{~h}$, the leaf discs were completely rehydrated and the trace of dark-induced increase of $\mathrm{F}_{0}^{\prime}$ showed an intermediate position - between those rehydrated for 1 and $5 \mathrm{~h}$. The presented results also clearly indicate that application of electron transport inhibitors of PGR5- (Fig. 5B), NDHdependent (Fig. 5C) CEFs, and PTOX-mediated electron transfer to $\mathrm{O}_{2}$ (Fig. 5D) strongly reduced the extent of post-illumination (dark) increase of $\mathrm{F}_{0}{ }^{\prime}$ during rehydration, compared to the same process occurring only in water. Considering that nonphotochemical (dark) increases of $F_{0}{ }^{\prime}$ have been attributed to the reduction of PQ pool related to PSI-dependent CEFs (Fisher and Kramer 2014), it is clear that the capacity for CEFs was greatly reduced in the presence of all electron transport inhibitors used.

\section{Discussion}

Resurrection plants are unique with their capability to tolerate desiccation up to the air-dried state. This capability is based on different mechanisms that include regulation of metabolism, subcellular reorganization, and stimulation of different antioxidant strategies (for review see Farrant et al. 2007, Morse et al. 2011, Giarola et al. 2017, Liu et al. 2019). As one of the most sensitive to environmental stress conditions, photosynthesis is considerably concerned under desiccation. The recovery from desiccation during plant rehydration is a complex and complicated process that allows consecutive restoration of main photochemical and biochemical processes leading to the recovery of net photosynthesis and full recovery of plants. The studies on resurrection plants have focused mainly on the protective mechanisms in the dehydration phase but no sufficient attention was paid to the characterization of rehydration processes (Giarola and Bartels 2015). While the later phase of the rehydration, when the plants regained almost full water content, is well studied, the earlier phase (first hours of rehydration) has remained out of the researchers' attention. Recently, we reported an analysis of the recovery process of $H$. rhodopensis plants desiccated as a result of drought or as a result of freezing comparing the recovery of photochemical activities of both photosystems and reorganization of main pigment-protein complexes (Georgieva et al. 2020). It has been shown that during rehydration PSI recovered earlier than PSII and that during the first hours of rehydration, prominent alterations in the energy transfer between photosynthetic complexes occurred.

In agreement with previous studies on resurrection plants (Huang et al. 2012, Zia et al. 2016), our experimental results also demonstrate that the inhibition of linear photosynthetic electron flow (LEF) in winter desiccated H. rhodopensis plants is associated with a substantial increase in the CEF. This is in full agreement with earlier transcriptomic data suggesting the presence of $\mathrm{NDH}$ complex in H. rhodopensis (Liu et al. 2018) and its possible role as a component of CEF (Mladenov et al. 2015). Upregulation of PSI-dependent CEF was suggested to play a significant role in photoprotection of the photosynthetic apparatus in the resurrection plants Paraboea rufescens (Huang et al. 2012) and Craterostigma pumilum (Zia et al. 2016) against drought/dehydration stress. However, the possible involvement of $\mathrm{CEF}$ and/or alternative electron flows during rehydration of desiccated plants, which determine the recovery of photosynthetic activities, has never been studied.

The experimental results presented in this study confirm our recent observation (Georgieva et al. 2020) 


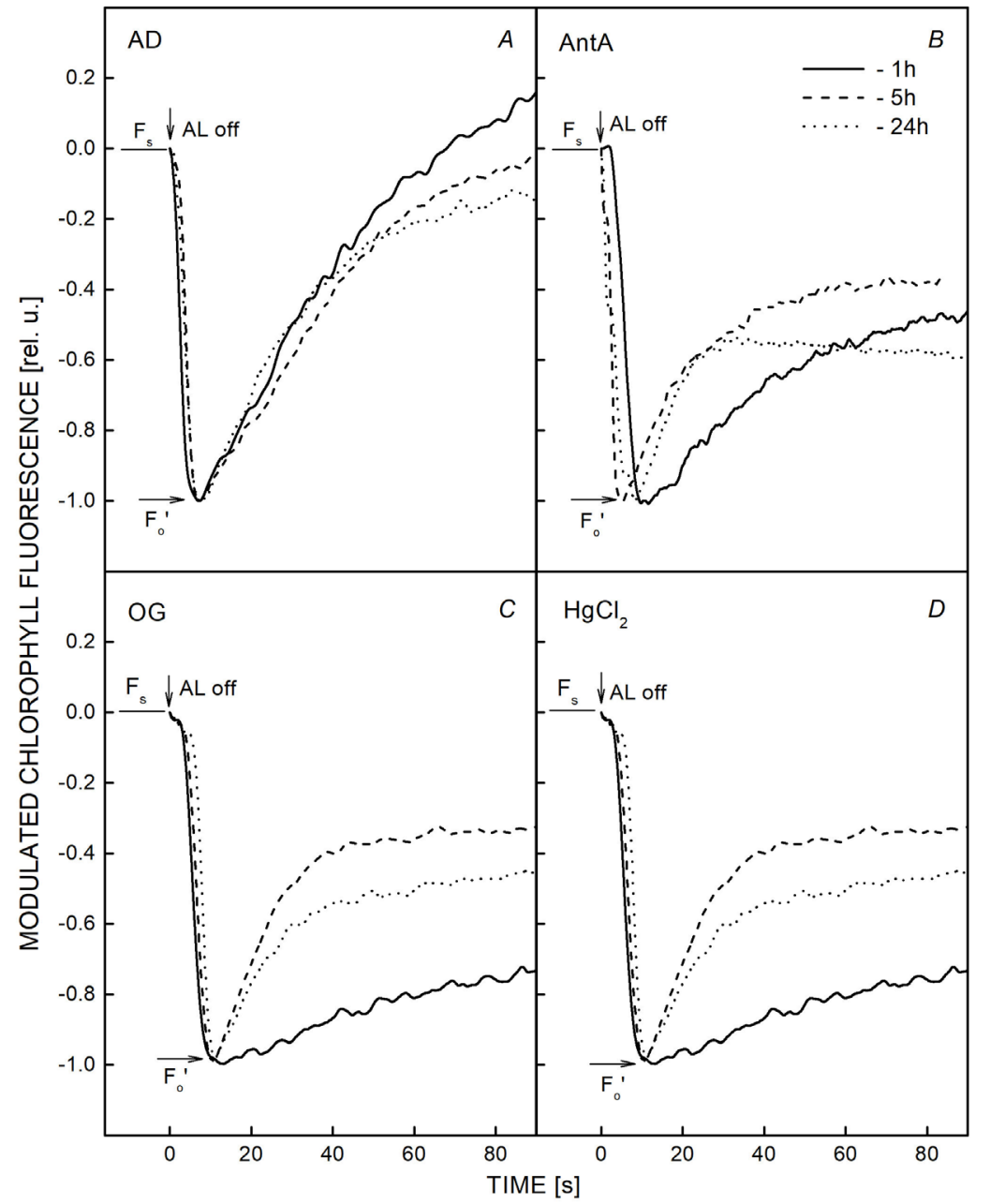

Fig. 5. Effects of rehydration time on postilluminational $\mathrm{F}_{0}{ }^{\prime}$ fluorescence increase after the actinic light $[\mathrm{AL}$ of $90 \mu \mathrm{mol}$ (photon) $\left.\mathrm{m}^{-2} \mathrm{~s}^{-1}, 6 \mathrm{~min}\right]$ was turned off in control, rehydrated in distilled water (AD) $(A)$ and dehydrated Haberlea rhodopensis leaf discs treated with electron transport inhibitors $10 \mu \mathrm{M}$ antimycin A (AntA) $(B), 20 \mu \mathrm{M}$ octyl gallate (OG) $(C)$, and $100 \mu \mathrm{M} \mathrm{HgCl}_{2}(D)$. The traces are averages from three measurements in two independent experiments $(n=6)$. that PSI photochemistry recovered much faster, reaching almost full recovery after 3 -h rehydration (Fig. 3A), compared to PSII activity which increased gradually and did not fully recover before $7 \mathrm{~h}$ of rehydration (Fig. 1A). In addition, the differential impact of the inhibitors of alternative electron pathways on the recovery dynamics of both photosystems demonstrates that PSI is more sensitive than PSII. Inhibition of PGR5-dependent CEF by AntA did not affect the recovery of PSII, but in its presence, the activity of PSI was restored only to $80 \%$ of that detected for leaf discs in AD. In addition, AntA drastically reduced the post-illuminational (dark) increase of $\mathrm{F}_{0}{ }^{\prime}$ (Fig. $5 B$ ) compared to plants rehydrating in water (Fig. $5 B$ ), thus clearly suggesting the involvement of the PGR5-dependent CEF pathway in the recovery from coldinduced desiccation. Similar, AntA-induced reduction of the post-illuminational $F_{0}{ }^{\prime}$ increase was reported earlier in cold-acclimated Arabidopsis plants (Ivanov et al. 2012). Elimination of PGR5-dependent CEF in AntA-treated maize discs led to the nearly complete prevention of $\mathrm{F}_{0}{ }^{\prime}$ increase, i.e., over-reduction of intersystem electron transport chain in cold-stressed plants (Savitch et al. 2011). PGR5-dependent CEF is dominant in $\mathrm{C}_{3}$ plants, especially under stressful conditions, such as drought, high light, or low $\mathrm{CO}_{2}$, and is essential for inducing $\mathrm{q}_{\mathrm{E}}$, thus determining its significance for photoprotection and preventing PSI from photodamage (Munekage et al. 2002, 2004; DalCorso et al. 2008).

A similar effect was observed when NDH-dependent CEF was blocked by rehydrating the leaf discs in the presence of $\mathrm{HgCl}_{2}$, while the recovery of PSII activity, although slightly reduced, followed the kinetics observed in $\mathrm{AD}$ (Fig. $1 A$ ), the recovery of PSI was even more inhibited compared to AntA-treated plants (Fig. $3 A$ ). This prevents over-reduction of $\mathrm{PQ}$ and, on the other hand, makes it difficult to extract electrons from the acceptor side of PSI, thus creating favorable conditions for PSII recovery, but preventing PSI recovery.

In $\mathrm{C}_{3}$ plants, the PGR5-dependent CEF is the major pathway and NDH-dependent is the minor and it has been shown that the loss of NDH-related CEF exhibited a minimal effect on the acclimation and growth of Arabidopsis (Shikanai and Yamamoto 2017). Although some differences between AntA and $\mathrm{HgCl}_{2}$ in respect to the time dependence of post-illumination transients were observed, the experimental results (Fig. 5) suggest that both AntA-sensitive (PGR5-dependent) and $\mathrm{HgCl}_{2-}$ sensitive (NDH-dependent) PSI-driven cyclic electron 
flows are involved in the rehydration process of desiccated H. rhodopensis plants.

The plastid terminal oxidase (PTOX) is involved not only in chlororespiration but also participates in alternative electron transport flows by transferring electrons from PQ directly to oxygen, thus serving as an effective electron transport sink (Cournac et al. 2000, McDonald et al. 2011). Inhibition of PTOX by OG significantly reduced reoxidation of PQ in cold-acclimated Arabidopsis plants (Ivanov et al. 2012). The importance of this electron transfer sink in alleviating various environmental stress conditions is supported by the upregulation of PTOX in light and heat-stressed Brassica (Díaz et al. 2007), saltstressed halophyte Thellundiella (Stepien and Johnson 2009), as well as in cold-acclimated Lodgepole pine (Savitch et al. 2010) and Arabidopsis thaliana (Ivanov et al. 2012). Our data support the involvement of PTOX-dependent electron transfer sink in the recovery of photosynthetic performance of both photosystems. Our observations clearly showed that rehydration in the presence of $\mathrm{OG}$, which inhibits the plastid terminal oxidase (PTOX), significantly reduced the $\mathrm{R}_{\mathrm{Fd}}$ values. In the view of current models, the functioning PGR5-dependent and $\mathrm{NDH}-d e p e n d e n t$ alternative electron transport pathways provided not only protection of over-reduction of electron transport chain but also the formation of proton motive force needed for ATP synthesis (Shikanai and Yamamoto 2017). In addition, the chlororespiration PTOX might be involved in the initiation of cyclic electron transfer reactions around PSI by regulating the redox state of the intersystem electron carriers (Peltier and Cournac 2002). The regulation of the redox state of $\mathrm{PQ}$ by chlororespiration could also affect the efficiency of NAD-independent cyclic pathways, such as the AntA-sensitive pathway. Recently, the involvement of cyclic electron flow around PSI has been identified as a major factor responsible for increased tolerance to desiccation of Porphyra yezoensis - during desiccation the PSI-driven electron flow was considerably enhanced and kept stimulated during rehydration (Gao and Wang 2012). The authors did not observe any effect of AntA on CEF in Porphyra yezoensis. The reason for this discrepancy can be that this species is quite different from higher plants and also due to the different experimental setup applied. Our data showed that the alternative cyclic electron pathways - PGR5-dependent and NDH-dependent - played a significant role in the recovery of PSI and PSII photochemical competence. By the effect of different inhibitors, it could be concluded that during the recovery of $H$. rhodopensis from desiccation, the NDH-dependent cyclic electron pathway played a more determining role in the recovery process of PSI and to the lesser extent of PSII. It has been reported that in response to environmental stress, the expression of the NDH complex and the activity of chlororespiration increased (Peltier and Cournac 2002, Savitch et al. 2011). In addition, it is worth noting that the influence of alternative flows is more pronounced in the first hours of rehydration. It has been shown that during desiccation the homoiochlorophyllous plant (Craterostigma pumilum) retain their photosynthetic apparatus at least in part via structural reorganization and rearrangement of pigment-protein complexes (Charuvi et al. 2015) that permitted them to recover photosynthetic activities during consecutive rehydration for $24 \mathrm{~h}$.

It is important to note that although the restricted PSII activity in winter-desiccated $H$. rhodopensis almost fully recovered during the rehydration (Fig. 1A), the capacity for $\mathrm{CEF}$ remained high and did not recover during the same period (Fig. 3B). Earlier studies reported a similarly slow recovery of CEF in Scots pine recovering from winter depression of photosynthesis (Ivanov et al. 2001) and during rehydration of desiccated Porphyra yezoensis (Gao and Wang 2012). We suggest that a high capacity for CEF is needed to supply extra ATP for rebuilding the photosynthetic apparatus during the recovery process until the capacity for $\mathrm{CO}_{2}$ assimilation serving as the major electron sink is recovered (Yang et al. 2020).

Conclusions: In green plants, the ATP/NADPH stoichiometry generated by the linear electron flow from water to $\mathrm{NADP}^{+}$is estimated to be 1.29 and generates 2.6 molecules of ATP per 2 molecules of NADPH (Sacksteder et al. 2000, Allen 2003), which is far from sufficient for sustained operation of the Calvin-Benson-Bassham (CBB) cycle requiring ATP/NADPH ratios of 1.5 and 1.75 for $\mathrm{CO}_{2}$ fixation and photorespiration, respectively, under optimal environmental conditions (Walker et al. 2016). It is clear that even under optimal conditions, LEF alone cannot generate enough ATP to support and sustain primary metabolism. Under severe environmental conditions, such as desiccation and especially in the early stages of recovery from desiccation in resurrection plants, when the LEF is still strongly reduced, an additional source of ATP is required. We believe that upregulation of both, partially redundant PGR5- and NDH-dependent CEFs reported in this study, can compensate the restricted LEF in providing the additional source of ATP (Yamori et al. 2011) and both alternative CEF pathways are indispensable in the process of recovery of Haberlea rhodopensis from freezing-induced desiccation (Fig. 6B). However, the elevated CEFs may cause over-reduction of the photosynthetic electron transport carriers including the primary electron acceptor of PSII $\left(\mathrm{Q}_{\mathrm{A}}\right)$. This would require activation of alternative oxygen-dependent electron pathways that can directly oxidize the PQ pool (Fig. 6, dark blue dotted arrows) thus avoiding its over-reduction. Our results demonstrate that the excess electrons can be diverted from the LEF and utilized through the enhanced PTOX-mediated alternative electron transfer to $\mathrm{O}_{2}$ (Fig. 6B, dark blue dotted line). The higher abundance of PTOX protein reported in various cold stressed/acclimated (Savitch et al. 2010, McDonald et al. 2011, Ivanov et al. 2012) plants supports this suggestion. In addition, the Mehler-ascorbate peroxidase pathway (WWC) (Asada 1999) has been also shown to effectively serve as an alternative electron sink (Fig. 6B) and this pathway may also be upregulated under low temperatures (Savitch et al. 2009). The enhanced capacity of both PTOX-dependent transfer to $\mathrm{O}_{2}$ and WWC when the 


\section{A Control}

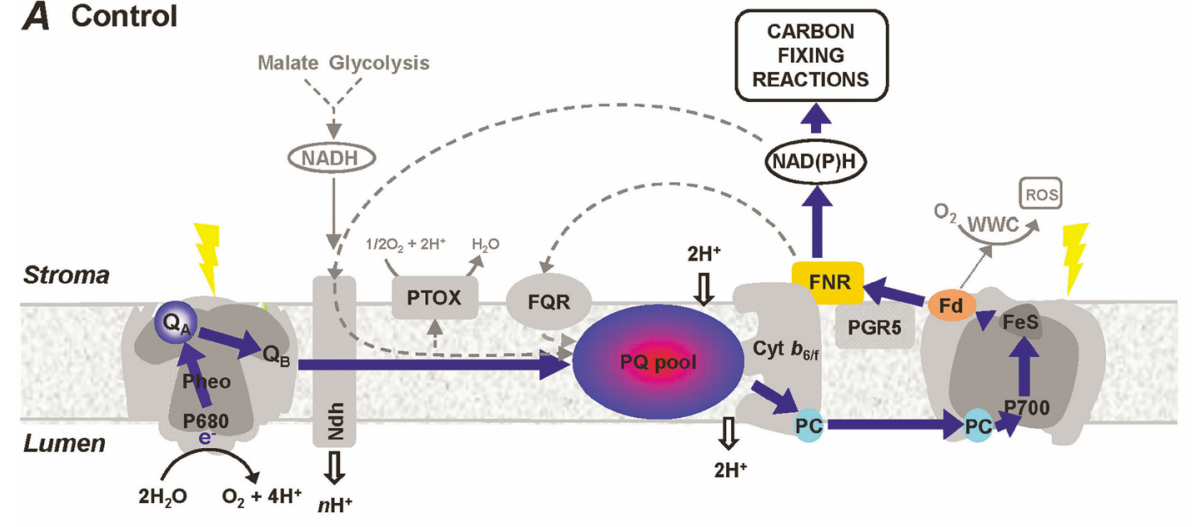

\section{B Winter dehydrated}

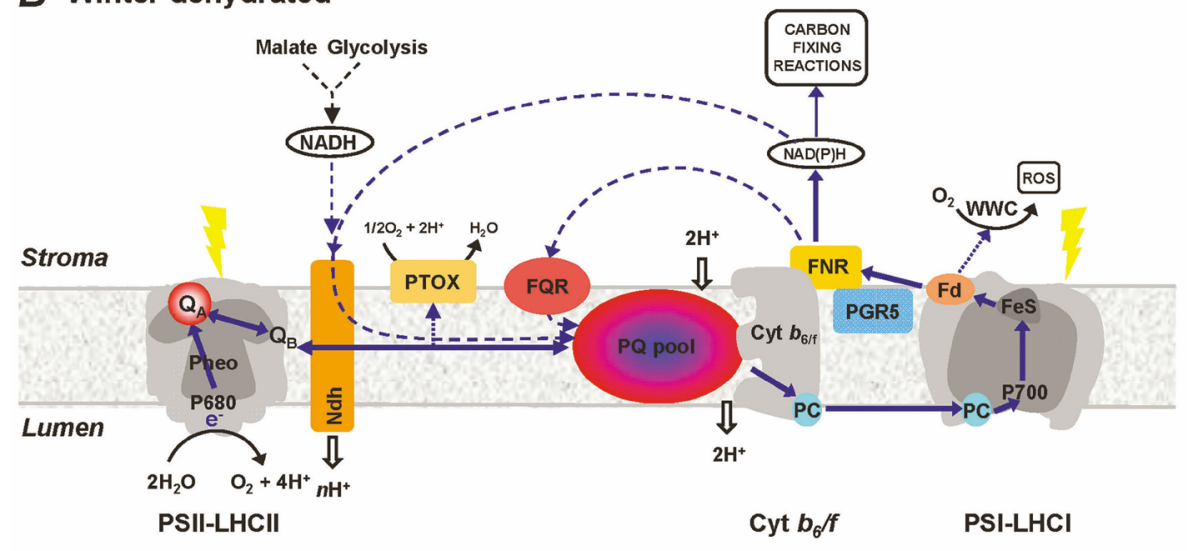

$\longrightarrow$ - Linear electron flow $-\longrightarrow \rightarrow$ - Cyclic electron flow $\quad \ldots . . . . \rightarrow-$ Alternative electron flow
- Oxidized $P Q$

Fig. 6. Simplified overview of electron transport pathways (dark blue arrows) within the thylakoid membranes of Haberlea rhodopensis acclimated to different growth conditions. $(A)$ During the growth and development of Haberlea rhodopensis plants under optimal temperature conditions the PQ pool remains preferentially oxidized because the rate of consumption of photosynthetic electrons through metabolic sinks (carbon-fixing reactions) keeps pace with the rate at which PSII undergoes charge separation to reduce the PQ pool. Under these conditions, the linear photosynthetic electron flow (dark blue solid arrows) from PSII (water splitting) to PSI (NADP ${ }^{+}$ generation) dominates. In addition, two partially redundant PSI-dependent cyclic electron transport (CET) pathways [blue (active) and gray (inactive) dashed arrows]: NDH-dependent and FQR(PGR5)-dependent might also be involved. (B) Exposure of Haberlea rhodopensis plants to winter conditions results in severe dehydration and reduction of PSII photochemistry, the linear electron flow, and $\mathrm{CO}_{2}$ assimilation (Fig. 1) compared to control plants. Under these conditions imposing strong limitations at the acceptor side of PSI, the excitation pressure over PSII increases, and the PQ pool becomes predominantly reduced (Fig. 2). The excess electrons not utilized by carbon metabolism can be recirculated by the NDH- and/or FQR-dependent PSI-dependent CEF pathways and/or diverted from the linear electron flow and utilized through alternative oxygen-dependent electron sinks. Our results demonstrate that both PSI-dependent cyclic electron transport pathways: NDH-dependent and FQR(PGR5)-dependent (blue dashed arrows) are upregulated in winter-dehydrated plants and during recovery from dehydration. In addition, the alternative PTOX-dependent electron donation to oxygen (blue dotted arrow) is also involved in diverting electrons from the photosynthetic electron transport chain under the same conditions.

requirement for PQ pool oxidization increases would guarantee the higher activity of CEFs (Miyake 2010, Ivanov et al. 2012). Thus, the employment of these alternative electron pathways as safety valves may play an important physiological role in balancing/regulating the linear/cyclic photosynthetic electron flows when the acceptor side of PSI is limited by low temperature, as in winter-desiccated Haberlea rhodopensis plants.

\section{References}

Allen J.F.: Cyclic, pseudocyclic and noncyclic photophosphorylation: new links in the chain. - Trends Plant Sci. 8: 15-19, 2003.

Arnon D.I., Allen M.B., Whatley F.R.: Photosynthesis by isolated chloroplasts. - Nature 174: 394-396, 1954.

Asada K.: The water-water cycle in chloroplasts: scavenging of active oxygens and dissipation of excess photons. - Annu. 
Rev. Plant Phys. 50: 601-639, 1999.

Asada K., Heber U., Schreiber U.: Pool size of electrons that can be donated to $\mathrm{P} 700^{+}$, as determined in intact leaves: donation to $\mathrm{P} 700^{+}$from stromal components via the intersystem chain. - Plant Cell Physiol. 33: 927-932, 1992.

Asada K., Heber U., Schreiber U.: Electron flow to the intersystem chain from stromal components and cyclic electron flow in maize chloroplasts, as determined in intact leaves by monitoring redox change of P700 and chlorophyll fluorescence. - Plant Cell Physiol. 34: 39-50, 1993.

Bewley J.D., Oliver M.J.: Desiccation tolerance in vegetative plant tissues and seeds: protein synthesis in relation to desiccation and a potential role for protection and repair mechanisms. In: Somero G.N., Osmond C.B., Bolis C.L. (ed.): Water and Life. Pp. 141-160. Springer, Berlin-Heidelberg 1992.

Charuvi D., Nevo R., Shimoni E. et al.: Photoprotection conferred by changes in photosynthetic protein levels and organization during dehydration of a homoiochlorophyllous resurrection plant. - Plant Physiol. 167: 1554-1565, 2015.

Cournac L., Josse E.M., Joët T. et al.: Flexibility in photosynthetic electron transport: a newly identified chloroplast oxidase involved in chlororespiration. - Philos. T. Roy. Soc. B 355: 1447-1454, 2000.

DalCorso G., Pesaresi P., Masiero S. et al.: A Complex containing PGRL1 and PGR5 is involved in the switch between linear and cyclic electron flow in Arabidopsis. - Cell 132: 273-285, 2008.

Demmig-Adams B., Adams III W.W., Barker D.H. et al.: Using chlorophyll fluorescence to assess the fraction of absorbed light allocated to thermal dissipation of excess excitation. Physiol. Plantarum 98: 253-264, 1996.

Díaz M., De Haro V., Muñoz R., Quiles M.J.: Chlororespiration is involved in the adaptation of Brassica plants to heat and high light intensity. - Plant Cell Environ. 30: 1578-1585, 2007.

Endo T., Mi H., Shikanai T., Asada K.: Donation of electrons to plastoquinone by $\mathrm{NAD}(\mathrm{P}) \mathrm{H}$ dehydrogenase and by ferredoxin-quinone reductase in spinach chloroplasts. - Plant Cell Physiol. 38: 1272-1277, 1997.

Farrant J.M.: Mechanisms of desiccation tolerance in angiosperm resurrection plants. - In: Jenks M.A., Wood A.J. (ed.): Plant Desiccation Tolerance. Pp. 51-90. Blackwell Publishing, Wallingford 2007.

Fisher N., Kramer D.M.: Non-photochemical reduction of thylakoid photosynthetic redox carriers in vitro: Relevance to cyclic electron flow around photosystem I? - BBABioenergetics 1837: 1944-1954, 2014.

Flores-Bavestrello A., Król M., Ivanov A.G. et al.: Two Hymenophyllaceae species from contrasting natural environments exhibit a homoiochlorophyllous strategy in response to desiccation stress. - J. Plant Physiol. 191: 82-94, 2016.

Gao S., Niu J., Chen W. et al:: The physiological links of the increased photosystem II activity in moderately desiccated Porphyra haitanensis (Bangiales, Rhodophyta) to the cyclic electron flow during desiccation and re-hydration. Photosynth. Res. 116: 45-54, 2013.

Gao S., Shen S., Wang G. et al.: PSI-driven cyclic electron flow allows intertidal macro-algae Ulva sp. (Chlorophyta) to survive in desiccated conditions. - Plant Cell Physiol. 52: 885-893, 2011.

Gao S., Wang G.: The enhancement of cyclic electron flow around photosystem I improves the recovery of severely desiccated Porphyra yezoensis (Bangiales, Rhodophyta). J. Exp. Bot. 63: 4349-4358, 2012.

Genty B., Briantais J.M., Baker N.R.: The relationship between the quantum yield of photosynthetic electron transport and quenching of chlorophyll fluorescence. - BBA-Gen. Subjects 990: 87-92, 1989.

Georgieva K., Mihailova G., Gigova L. et al.: The role of antioxidant defense in freezing tolerance of resurrection plant Haberlea rhodopensis. - Physiol. Mol. Biol. Pla. 27: 11191133, 2021.

Georgieva K., Mihailova G., Velitchkova M., Popova A.: Recovery of photosynthetic activity of resurrection plant Haberlea rhodopensis from drought- and freezing-induced desiccation. - Photosynthetica 58: 911-921, 2020.

Georgieva K., Rapparini F., Bertazza G. et al.: Alterations in the sugar metabolism and in the vacuolar system of mesophyll cells contribute to the desiccation tolerance of Haberlea rhodopensis ecotypes. - Protoplasma 254: 193-201, 2017.

Giarola V., Bartels D.: What can we learn from the transcriptome of the resurrection plant Craterostigma plantagineum? Planta 242: 427-434, 2015.

Giarola V., Hou Q., Bartels D.: Angiosperm plant desiccation tolerance: hints from transcriptomics and genome sequencing.Trends Plant Sci. 22: 705-717, 2017.

Huang W., Yang S.-J., Zhang S.-B. et al.: Cyclic electron flow plays an important role in photoprotection for the resurrection plant Paraboea rufescens under drought stress. - Planta 235: 819-828, 2012.

Hüner N.P.A., Dahal K., Bode R. et al.: Photosynthetic acclimation, vernalization, crop productivity and 'the grand design of photosynthesis'. - J. Plant Physiol. 203: 29-43, 2016.

Ivanov A.G., Morgan R.M., Gray G.R. et al.: Temperature/ light dependence development of selective resistance to photoinhibition of photosystem I. - FEBS Lett. 430: 288-292, 1998.

Ivanov A.G., Rosso D., Savitch L.V. et al:: Implications of alternative electron sinks in increased resistance of PSII and PSI photochemistry to high light stress in cold acclimated Arabidopsis thaliana. - Photosynth. Res. 113: 191-206, 2012.

Ivanov A.G., Sane P.V., Zeinalov Y. et al.: Photosynthetic electron transport adjustments in overwintering Scots pine (Pinus sylvestris L.). - Planta 213: 575-585, 2001.

Klughammer C., Schreiber U.: Analysis of light-induced absorbency changes in the near-infrared spectral region. 1. Characterization of various components in isolated chloroplasts. - Z. Naturforsch. C 46: 233-244, 1991.

Lichtenthaler H.K., Miehé J.A.: Fluorescence imaging as a diagnostic tool for plant stress. - Trends Plant Sci. 2: 316$320,1997$.

Lichtenthaler H.K., Rinderle U.: The role of chlorophyll fluorescence in the detection of stress conditions in plants. CRC Crit. Rev. Anal. Chem. 19: S29-S85, 1988.

Liu J., Moyankova D., Djilianov D., Deng X.: Common and specific mechanisms of desiccation tolerance in two Gesneriaceae resurrection plants. Multiomics evidences. Front. Plant Sci. 10: 1067, 2019.

Liu J., Moyankova D., Lin C.-T. et al.: Transcriptome reprogramming during severe dehydration contributes to physiological and metabolic changes in the resurrection plant Haberlea rhodopensis. - BMC Plant Biol. 18: 351, 2018.

Losciale P., Oguchi R., Hendrickson L. et al.: A rapid, wholetissue determination of the functional fraction of PSII after photoinhibition of leaves based on flash-induced P700 redox kinetics. - Physiol. Plantarum 132: 23-32, 2008.

Mano J., Miyake C., Schreiber U., Asada K.: Photoactivation of electron flow from NADPH to plastoquinone in spinach chloroplasts. - Plant Cell Physiol. 36: 1589-1598, 1995.

Maxwell P.C., Biggins J.: Role of cyclic electron transport in photosynthesis as measured by the photoinduced turnover of 
P700 in vivo. - Biochemistry 15: 3975-3981, 1976.

McDonald A.E., Ivanov A.G., Bode R. et al.: Flexibility in photosynthetic electron transport: the physiological role of plastoquinol terminal oxidase (PTOX). - BBA-Bioenergetics 1807: 954-967, 2011.

Mihailova G., Solti Á., Sárvári É. et al.: Freezing tolerance of photosynthetic apparatus in the homoiochlorophyllous resurrection plant Haberlea rhodopensis. - Environ. Exp. Bot. 178: 104157, 2020

Miyake C.: Alternative electron flows (water-water cycle and cyclic electron flow around PSI) in photosynthesis: molecular mechanisms and physiological functions. - Plant Cell Physiol. 51: 1951-1963, 2010.

Mladenov P., Finazzi G., Bligny R. et al.: In vivo spectroscopy and NMR metabolite fingerprinting approaches to connect the dynamics of photosynthetic and metabolic phenotypes in resurrection plant Haberlea rhodopensis during desiccation and recovery. - Front. Plant Sci. 6: 564, 2015.

Moore J.P., Le N.T., Brandt W.F. et al.: Towards a systems-based understanding of plant desiccation tolerance. - Trends Plant Sci. 14: 110-117, 2009.

Morse M., Rafudeen M.S., Farrant J.M.: An overview of the current understanding of desiccation tolerance in the vegetative tissues of higher plants. - In: Turkan I. (ed.): Advances in Botanical Research. Vol. 57. Pp. 319-347. Elsevier, Amsterdam 2011.

Munekage Y., Hashimoto M., Miyake C. et al.: Cyclic electron flow around photosystem I is essential for photosynthesis. Nature 429: 579-582, 2004.

Munekage Y., Hojo M., Meurer J. et al.: PGR5 is involved in cyclic electron flow around photosystem I and is essential for photoprotection in Arabidopsis. - Cell 110: 361-371, 2002.

Peltier G., Cournac L.: Chlororespiration. - Annu. Rev. Plant Biol. 53: 523-550, 2002.

Ravenel J., Peltier G., Havaux M.: The cyclic electron pathways around photosystem I in Chlamydomonas reinhardtii as determined in vivo by photoacoustic measurements of energy storage. - Planta 193: 251-259, 1994.

Sacksteder C.A., Kanazawa A., Jacoby M.E., Kramer D.M.: The proton to electron stoichiometry of steady-state photosynthesis in living plants: a proton-pumping Q cycle is continuously engaged. - P. Natl. Acad. Sci. USA 97: 14283-14288, 2000.

Sárvári E., Mihailova G., Solti Á. et al.: Comparison of thylakoid structure and organization in sun and shade Haberlea rhodopensis populations under desiccation and rehydration. J. Plant Physiol. 171: 1591-1600, 2014.

Savitch L.V., Ivanov A.G., Gudynaite-Savitch L. et al.: Effects of low temperature stress on excitation energy partitioning and photoprotection in Zea mays. - Funct. Plant Biol. 36: 37-49, 2009.

Savitch L.V., Ivanov A.G., Gudynaite-Savitch L. et al.: Cold stress effects on PSI photochemistry in Zea mays: differential increase of FQR-dependent cyclic electron flow and functional implications. - Plant Cell Physiol. 52: 1042-1054, 2011.

Savitch L.V., Ivanov A.G., Krol M. et al.: Regulation of energy partitioning and alternative electron transport pathways during cold acclimation of Lodgepole pine is oxygen dependent. Plant Cell Physiol. 51: 1555-1570, 2010.

Shikanai T.: Cyclic electron transport around photosystem I: Genetic approaches. - Annu. Rev. Plant Biol. 58: 199-217, 2007.

Shikanai T., Endo T., Hashimoto T. et al.: Directed disruption of the tobacco $n d h B$ gene impairs cyclic electron flow around photosystem I. - P. Natl. Acad. Sci. USA 95: 9705-9709, 1998.

Shikanai T., Yamamoto H.: Contribution of cyclic and pseudocyclic electron transport to the formation of proton motive force in chloroplasts. - Mol. Plant 10: 20-29, 2017.

Stepien P., Johnson G.N.: Contrasting responses of photosynthesis to salt stress in the glycophyte Arabidopsis thaliana and the halophyte Tellungiella halophila. Role of the plastid terminal oxidase as an alternative electron sink. - Plant Physiol. 149: $1154-1165,2009$.

Tagawa K., Tsujimoto H.Y., Arnon D.I.: Role of chloroplast ferredoxin in the energy conversion process of photosynthesis. - P. Natl. Acad. Sci. USA 49: 567-572, 1963.

van Kooten O., Snel J.F.H.: The use of chlorophyll fluorescence nomenclature in plant stress physiology. - Photosynth. Res. 25: 147-150, 1990.

Walker B.J., VanLoocke A., Bernacchi C.J., Ort D.R.: The costs of photorespiration to food production now and in the future. - Annu. Rev. Plant Biol. 67: 107-129, 2016.

Yamori W., Sakata N., Suzuki Y. et al.: Cyclic electron flow around photosystem I via chloroplast $\mathrm{NAD}(\mathrm{P}) \mathrm{H}$ dehydrogenase (NDH) complex performs a significant physiological role during photosynthesis and plant growth at low temperature in rice. - Plant J. 68: 966-976, 2011.

Yang Q., Blanco N.E., Hermida-Carrera C. et al.: Two dominant boreal conifers use contrasting mechanisms to reactivate photosynthesis in the spring. - Nat. Commun. 11: 128, 2020.

Zia A., Walker B.J., Oung H.M.O. et al.: Protection of the photosynthetic apparatus against dehydration stress in the resurrection plant Craterostigma pumilum. - Plant J. 87: 664-680, 2016.

(C) The authors. This is an open access article distributed under the terms of the Creative Commons BY-NC-ND Licence. 